\title{
Wie verändert die COVID-19-Pandemie unsere Sexualitäten? Eine Übersicht medialer Narrative im Frühjahr 2020
}

\author{
How is the COVID-19 Pandemic Changing our Sexualities? \\ An Overview of Media Narratives in Spring 2020
}

\author{
Autor_innen \\ Nicola Döring und Roberto Walter
}

Institut

Institut für Medien und Kommunikationswissenschaft, Technische Universität Ilmenau

\section{Schlüsselwörter}

Coronavirus; COVID-19; Inhaltsanalyse; mediale

Darstellungen von Sexualität; Sexualität

\section{Keywords}

content analysis; Coronavirus; COVID-19; media

representations of sexuality; sexuality

\section{Bibliografie}

DOI https://doi.org/10.1055/a-1165-7760

Z Sexualforsch 2020; 33: 65-75

(c) Georg Thieme Verlag KG Stuttgart · New York

ISSN 0932-8114

\section{Korrespondenzadresse}

Prof. Dr. phil. Nicola Döring

Technische Universität Ilmenau

Institut für Medien und Kommunikationswissenschaft

Ehrenbergstr. 29 (EAZ 2217)

98693 Ilmenau

Nicola.Doering@tu-ilmenau.de

\section{ZUSAMMENFASSUNG}

Einleitung Anlässlich der COVID-19-Pandemie wurden ab März 2020 in Deutschland und vielen anderen Ländern weitreichende Infektionsschutzmaßnahmen verhängt. Deren Auswirkungen auf Individuen und Gesellschaft waren sofort Gegenstand intensiver medialer Debatten.

Forschungsziele Vor diesem Hintergrund verfolgt die vorliegende Studie das Ziel, die medialen Narrative speziell zu sexualitätsbezogenen Veränderungen durch die COVID-19Pandemie herauszuarbeiten.

Methoden Dazu wurde eine Stichprobe von N=305 massenmedialen Beiträgen aus dem Februar und März 2020 hinsichtlich 1) der behandelten Aspekte von Sexualität (z. B. Partnersex, Solosex), 2) der angebotenen Corona-Sex-Narrative (z. B. MehrPartnersex-Narrativ; Weniger-Partnersex-Narrativ) sowie 3) der Meta-Narrative (z. B. Krise als Chance, Krise als Risiko, Krise als Chance und Risiko zugleich) analysiert. Zudem wurden exemplarisch Beiträge aus Sozialen Medien untersucht. Die Studie folgt dem Open Science Ansatz: Stichprobe, Codebuch, Reliabilitätskoeffizienten und Datensatz sind über den Server der Open Science Foundation zugänglich (https://osf.io/ew6t3/).

Ergebnisse Es zeigte sich, dass in den Massenmedien Veränderungen beim Partnersex und Solosex sowie hinsichtlich verschiedener Aspekte sexueller und reproduktiver Gesundheit und Rechte auf der Agenda standen. Dabei wurden für Partnersex teilweise und für Solosex durchgängig Corona-SexNarrative angeboten, die eine Verbesserung der Situation in dem Sinne behaupten, dass es zu mehr und zu lustvollerem Sex kommt. Gleichzeitig gingen die problembezogenen CoronaSex-Narrative fast durchgängig von einer Verschlimmerung der Lage aus, etwa einem Mehr an sexualisierter häuslicher Gewalt, einem Mehr an Zugangshürden zum Schwangerschaftsabbruch, einem Mehr an Ungewissheiten bei Schwangerschaft und Geburt, einem Mehr an ökonomisch existenzbedrohlichen Lagen in der Sexarbeit und einem Mehr an Diskriminierung von LGBTIQ-Personen.

Schlussfolgerung Im medialen Diskurs über sexualitätsbezogene Auswirkungen der COVID-19-Pandemie zeigten sich zwei auffällige Tendenzen: Eine sehr sexpositive, kommerzfreundliche bis geradezu glorifizierende Würdigung von Solosex und Onlinesex sowie eine starke Sensibilisierung für bestimmte Einschränkungen der sexuellen und reproduktiven Selbstbestimmung. Es bleibt unklar, inwiefern die medialen Corona-Sex-Narrative tatsächliche Veränderungen umfassend und akkurat abbilden, da dazu empirische Daten fehlen.

\section{ABSTRACT}

Introduction On the occasion of the COVID-19 pandemic, far-reaching infection control measures were imposed in Germany and many other countries from March 2020. Their effects on individuals and society were immediately the subject of intense media debate.

Objectives Against this background, the present study aims to elaborate the media narratives specifically on sexuality-related changes caused by the COVID-19 pandemic.

Methods To this end, a sample of $N=305$ mass media contributions from February and March 2020 was analyzed with regard to 1 ) the aspects of sexuality dealt with (e.g., partnered sex, solo sex), 2) the corona-sex-narratives offered (e.g., more-partnered-sex-narrative; less-partnered-sex-narrative), and 3) the meta-narratives represented (e.g., crisis as opportunity, 
crisis as risk, crisis as opportunity and risk at the same time). In addition, selected contributions from social media were examined. The study follows the Open Science approach: sample, codebook, reliability coefficients and data set are accessible via the server of the Open Science Foundation (https://osf.io/ew6t3/).

Results It was shown that changes regarding partnered sex and solo sex as well as various issues of sexual and reproductive health and rights were on the mass media agenda. Corona-sex-narratives were offered in part for partnered sex and throughout for solo sex, which claim that the crisis has improved the situation in the sense that more and more pleasurable sex occurs. At the same time, the problem-related Corona-sex-narratives generally assumed that the situation has worsened, for example, that there is an increase in sexualized domestic violence, in access barriers to abortion, in uncertainties regarding pregnancy and birth, in threats to economic survival in sex work and in discrimination against LGBTIQ persons.

Conclusion In the media discourse on sexuality-related effects of the COVID-19 pandemic, two striking tendencies became apparent: A very sex-positive, commercially friendly to almost glorifying appreciation of solo and online sex and a strong sensitization for certain restrictions of sexual and reproductive self-determination. It remains unclear to what extent the Corona-sex-narratives comprehensively and accurately depict actual changes, as empirical data are lacking.
Die Coronavirus Disease 2019 (COVID-19) ist seit Dezember 2019 bekannt und verbreitete sich Anfang 2020 rapide weltweit. Diese neue Infektionskrankheit geht mit Grippesymptomen einher und führt bei schweren Verläufen zu Lungenentzündung, Lungenversagen und Tod (RKI 2020). Die WHO sprach ab dem 11. März 2020 von einer Pandemie (WHO 2020). Oberstes Ziel war es seitdem, die Verbreitung des für die Krankheit verantwortlichen - durch Tröpfcheninfektion leicht übertragbaren - neuartigen Coronavirus (SARS-CoV-2) zu verlangsamen. Dazu wurde in Deutschland wie auch in vielen anderen Ländern - das öffentliche Leben ab Mitte März stark eingeschränkt durch Schließung aller nicht systemrelevanten Einrichtungen, Kontakt- und Versammlungsverbote, Reiseund Ausgangseinschränkungen. Im Frühjahr 2020 befand sich mehr als die Hälfte der Weltbevölkerung im Lockdown (Sandfort 2020).

Welchen Einfluss hat die historische Ausnahmesituation der Corona-Krise, die mit wochenlanger häuslicher Isolation, fehlenden Arbeits-, Rückzugs- und Freizeitmöglichkeiten sowie gesundheitlicher und ökonomischer Existenzbedrohung einhergeht, auf das sexuelle Verhalten und Erleben? Diese Frage stellten sich nicht nur Fachkräfte aus dem Bereich der sexuellen Gesundheit, Bildung und Sexualforschung (z. B. Alpalhão und Filipe 2020; Hall et al. 2020; Hussein 2020). Diese Frage wurde seit März 2020 auch in der breiten Öffentlichkeit intensiv verhandelt. Denn wenn eines während der Corona-Krise nicht stillstand, dann war es der mediale Diskurs. Er wurde im Gegenteil sogar angeheizt durch die Krise: Die Ausnahmesituation erhöhte den allgemeinen Informations- und Kommunikationsbedarf, die Pandemie führte zur „Infodemie“ (Nielsen et al. 2020): In Massenmedien und Sozialen Medien wurde praktisch rund um die Uhr über die COVID-19-Pandemie debattiert - und sexuelle Aspekte waren dabei durchaus im Fokus.

Dass im Zusammenhang mit der Corona-Krise auch sexuelle Themen so schnell auf die Agenda rückten, ist erfreulich. Denn Fragen der sexuellen und reproduktiven Gesundheit und Rechte sowie des sexuellen Wohlbefindens sollten auch (und vielleicht: gerade) in Krisenzeiten ernst genommen werden, da sie eng mit der Gesamtgesundheit und Lebensqualität zusammenhängen. Hier können Medien aufklärend und unterstützend wirken (Döring 2015: Kap. 3). Gleichzeitig ist zu beachten, dass der mediale Diskurs um „Sex und Corona“ natürlich auch der alten Regel Sex sells folgt (Streitmatter 2004): Das Thema weckt Aufmerksamkeit und erhöht Klickzahlen, speziell wenn es reißerisch aufbereitet ist. Vor diesem Hintergrund verfolgt die vorliegende Studie das Ziel einer ersten Bestandsaufnahme und Einordnung medialer Narrative zu sexualitätsbezogenen Auswirkungen der COVID-19-Pandemie. Dabei sollen drei Forschungsfragen beantwortet werden:

- F1: Welche Aspekte von Sexualität werden im Zusammenhang mit der COVID-19-Pandemie in den Medien behandelt (z. B. Partnersex, Solosex, sexuelle Gewalt)?

- F2: Welche Narrative zur Veränderung der einzelnen Aspekte von Sexualität durch die COVID-19-Pandemie werden in den Medien angeboten (z. B. Mehr-Partnersex-Narrativ, WenigerPartnersex-Narrativ)?

- F3: Welche Meta-Narrative zur übergeordneten Bewertung der Veränderungen von Sexualität durch die COVID-19-Pandemie sind in den Medien zu finden (z. B. Krise als Chance, Krise als Risiko, Krise als Chance und Risiko zugleich)?

\section{Methoden}

Zur Beantwortung der Forschungsfragen wurde eine quantitative Medieninhaltsanalyse einer Stichprobe von massenmedialen Online-Beiträgen realisiert. Die Studie folgt dem Open Science Ansatz: Stichprobe, Codebuch, Reliabilitätskoeffizienten und Datensatz sind über den Server der Open Science Foundation zugänglich (https://osf.io/ew6t3/).

\section{Stichprobe}

Für die Stichprobenziehung wurden Anfang April 2020 mit der Suchmaschine Google alle öffentlich kostenfrei verfügbaren deutschsprachigen Online-Artikel aus Massenmedien zum Thema Sexualität und Corona identifiziert, indem das Suchwort „Corona“ mit sexualitätsbezogenen Suchbegriffen (z. B. „Sexualität“, „Sex“, "Schwangerschaft“, „Masturbation“, „sexuelle Gewalt“ usw.) kombiniert wurde. Bei dem Sample handelt es sich somit um eine gezielte Auswahl (purposive sampling; Döring und Bortz 2016: Kap. 9), die durch die Wahl der Suchbegriffe sowie den Google-Algorithmus beeinflusst ist.

Die erzielte Stichprobe enthält $N=305$ Medienbeiträge, die zwischen dem 18. Februar 2020 und dem 31. März 2020 in Medien aus Deutschland, Österreich und der Schweiz veröffentlicht wurden, 
wobei die allermeisten Beiträge (84.9\%) in der zweiten März-Hälfte nach Verhängung der Corona-Maßnahmen erschienen. Bei den massenmedialen Beiträgen im Sample handelt es sich um OnlinePressebeiträge sowie um Online-Begleittexte zu Radio- und Fernsehprogrammen. Das Sample ist heterogen und umfasst Beiträge aus der Boulevardpresse (z. B. „Die BILD“) ebenso wie der Qualitätspresse (z. B. „Die ZEIT“), von öffentlich-rechtlichen (z. B. ARD) ebenso wie von privaten (z. B. RTL) Rundfunksendern, von konservativ (z. B. „FAZ“) ebenso wie von progressiv (z. B. „taz“) ausgerichteten Medien, von generellen Publikumsmedien ebenso wie von zielgruppen- und themenspezifischen Medien (z. B. „queer.de“, „evangelisch.de“). Ergänzend zu dem Sample der massenmedialen Beiträge wurden auch einzelne Beiträge aus Sozialen Medien (YouTube, Facebook, Twitter) in die Untersuchung einbezogen.

\section{Instrument und Datenerhebung}

Die Datenerhebung erfolgte durch Codierung aller Medienbeiträge im Sample anhand eines überwiegend induktiv entwickelten Codebuchs (Reliabilitätstest mit zwei unabhängigen Codierenden, durchschnittlicher Koeffizient Cohens Kappa = .87) mit den zentralen Oberkategorien 1) Aspekte der Sexualität, 2) Corona-Sex-Narrative und 3) Corona-Sex-Meta-Narrative. Ein Narrativ bezieht sich dabei auf Aussagen über die Veränderung einer Situation in der Zeit (Müller 2019; Prince 1981): Ein Ausgangszustand (hier: Sexualität vor der COVID-19-Pandemie) wird in der medialen Darstellung durch ein transformatives Ereignis (hier: COVID-19-Pandemie mit weitreichenden Infektionsschutzmaßnahmen) in einen neuen Zustand überführt (hier: Sexualität in/nach der COVID-19-Pandemie). Die narrative Struktur „Ausgangssituation-Ereignis-Endsituation“ wird in der vorliegenden Studie im Rahmen einer inhaltsbezogenen Form der narrativen Analyse erfasst (Robertson 2017).

\section{Datenanalyse und Interpretation}

Die Datenanalyse mit SPSS (Version 24) umfasste nur deskriptivstatistische Häufigkeitsanalysen, da keine Hypothesen zur Prüfung vorlagen. Die Plausibilität der Narrative wurde post hoc anhand von Theorien und Befunden zu vergleichbaren Situationen interpretativ eingeordnet.

\section{Übersicht der Corona-Sex-Narrative}

Es zeigte sich, dass der mediale Diskurs im Frühjahr 2020 Corona-bedingte Veränderungen beim Partnersex (43.9\% der Medienbeiträge im Sample) sowie beim Solosex (35.7\%) umfassend thematisierte und dafür jeweils vier verschiedene Veränderungs-Narrative anbot (siehe - Tab. 1). Die häufigsten Partnersex-Narrative beschrieben oder forderten im Zuge des Infektionsschutzes weniger unverbindlichen Partnersex (18.7\%) und dafür mehr Telefon- und Onlinesex (21.6\%). Die häufigsten Solosex-Narrative beschrieben oder empfahlen während der Corona-bedingt vermehrten Selbstbefriedigung die Nutzung von mehr Sextoys (14.4\%) und mehr Pornografie (13.4\%). Der mediale Diskurs war aber auch stark durch sexualitätsbezogene Problem-Narrative geprägt: $68.5 \%$ der untersuchten Medienbeiträge thematisierten sexualisierte häusliche Gewalt, Schwangerschaftsabbruch, Schwangerschaft und Geburt, Sexarbeit sowie LGBTIQ-Personen und boten für diese thematisierten Aspekte von Sexualität überwiegend Narrative der Problemverschärfung an (siehe > Tab. 1).
Deswegen ist es auch nicht verwunderlich, dass die Mehrzahl der Medienbeiträge (57.8\%) die COVID-19-Pandemie auf der Meta-Ebene als Risiko für die sexuellen Verhältnisse einstufte. Gleichzeitig sahen aber auch relativ viele Beiträge (29.4\%) Chancen für das Sexualleben und vermittelten somit ein positives Meta-Narrativ (siehe $>$ Tab. 1). Die einzelnen Corona-Sex-Narrative werden im Folgenden anhand von Zitaten aus dem Medienmaterial genauer vorgestellt.

> Tab. 1 Übersicht der Corona-Sex-Narrative im Frühjahr 2020.

\begin{tabular}{|c|c|c|}
\hline & $\begin{array}{l}\text { Aspekte von } \\
\text { Sexualität \% }\end{array}$ & $\begin{array}{l}\text { Corona-Sex- } \\
\text { Narrative \% }\end{array}$ \\
\hline Partnersex-Narrative & 43.9 & \\
\hline Mehr Beziehungssex plus Babyboom & & 4.9 \\
\hline $\begin{array}{l}\text { Erhöhte Geburtenrate und erhöhte } \\
\text { Scheidungsrate }\end{array}$ & & 2.6 \\
\hline Weniger unverbindlicher Partnersex & & 18.7 \\
\hline Mehr Telefonsex und mehr Onlinesex & & 21.6 \\
\hline Solosex-Narrative & 35.7 & \\
\hline Mehr Masturbation & & 11.5 \\
\hline Mehr Sextoys & & 14.4 \\
\hline Mehr Pornografie & & 13.4 \\
\hline Neues Genre Coronavirus-Pornos & & 5.9 \\
\hline Sexualbezogene Problem-Narrative & 68.5 & \\
\hline Sexualisierte häusliche Gewalt & 12.5 & \\
\hline Mehr sexualisierte häusliche Gewalt & & 11.8 \\
\hline Schwangerschaftsabbruch & 5.9 & \\
\hline $\begin{array}{l}\text { Mehr Zugangshürden zum } \\
\text { Schwangerschaftsabbruch }\end{array}$ & & 5.2 \\
\hline Schwangerschaft und Geburt & 14.1 & \\
\hline $\begin{array}{l}\text { Mehr Ungewissheiten bei } \\
\text { Schwangerschaft und Geburt }\end{array}$ & & 11.8 \\
\hline Sexarbeit & 34.1 & \\
\hline $\begin{array}{l}\text { Mehr existenzbedrohliche Lagen in der } \\
\text { Sexarbeit }\end{array}$ & & 18.4 \\
\hline LGBTIQ-Personen & 6.9 & \\
\hline $\begin{array}{l}\text { Mehr Diskriminierung von LGBTIQ- } \\
\text { Personen }\end{array}$ & & 3.9 \\
\hline \multicolumn{3}{|l|}{ Meta-Narrative } \\
\hline Krise als Chance & & 29.4 \\
\hline Krise als Risiko & & 57.8 \\
\hline Krise als Chance und Risiko zugleich & & 12.8 \\
\hline
\end{tabular}

Anmerkung. Die Prozentwerte bei den Aspekten der Sexualität und den Corona-Sex-Narrativen berichten jeweils das Vorkommen im Vergleich zur Basis aller $N=305$ Medienbeiträge. Diese Werte summieren sich nicht zu $100 \%$ auf, da mehrere Aspekte und Narrative pro Medienbeitrag codiert werden konnten. Die Prozentwerte der Meta-Narrative berichten das Vorkommen im Vergleich zur Basis der $N=282$ Medienbeiträge mit Corona-Sex-Narrativen. Diese Werte summieren sich zu $100 \%$ auf, da immer genau ein Meta-Narrativ pro Medienbeitrag codiert wurde. 


\section{Partnersex-Narrative}

Es konnten vier Corona-Sex-Narrative identifiziert werden, die sich auf Veränderungen des Partnersex durch die Corona-Krise beziehen. Mit Partnersex sind sexuelle Interaktionen mit mindestens einer weiteren Person gemeint. Partnersex kann innerhalb und außerhalb fester Paarbeziehungen stattfinden.

\section{Das Mehr-Beziehungssex-plus-Babyboom-Narrativ}

Das Mehr-Beziehungssex-plus-Babyboom-Narrativ geht davon aus, dass häuslicher Rückzug und eingeschränkte Arbeits-, Freizeit- und Konsummöglichkeiten während der Corona-Maßnahmen eine Zunahme der sexuellen Aktivitäten bei zusammenlebenden Paaren bewirken: Man hat mehr Zeit gemeinsam zu Hause, somit mehr Gelegenheiten für die körperliche Liebe, was sich neun Monate später - geplant oder ungeplant - dann auch in einem Babyboom niederschlagen wird:

- „Mehr Corona, mehr Sex, mehr Babys“ verkündet die Tageszeitung „Volksstimme.de“.

- Der Radiosender YouFM prognostiziert ebenfalls einen Babyboom und zitiert die Beziehungsexpertin Nina Deißler mit der einschlägigen Corona-Parole „vögeln statt hamstern“.

- Das Online-Magazin „Miss.at“ hat sogar schon eine Bezeichnung für die Generation der Corona-Babys parat, nicht „Millenials“ sollen sie heißen, sondern „Coronials“.

- Das Redaktionsnetzwerk Deutschland verweist auf die während der Corona-Krise deutlich gestiegene Kondomnachfrage und spekuliert, dass mögliche Lieferengpässe bei Kondomen ebenfalls zum Babyboom beitragen könnten.

Das Mehr-Beziehungssex-plus-Babyboom-Narrativ ist dabei nicht neu. Es ist schon fast sprichwörtlich, dass genau neun Monate nach einem größeren Stromausfall die Geburtenzahl deutlich ansteigen soll. Denn ohne Strom würden sich die Menschen plötzlich verstärkt einander zuwenden und im Kerzenschein der körperlichen Liebe nachgehen, so die romantische These. Tatsächlich wurde der sogenannte Blackout Baby Boom, von dem die Zeitungen auf der Basis anekdotischer Beispiele immer wieder gern berichten, anhand systematischer empirischer Daten aber nie nachgewiesen und wird inzwischen eher als „sexualbezogene urbane Legende“ (Brunvand 1993) eingestuft: Die Geburtenrate neun Monate nach der Ausnahmesituation eines historischen Stromausfalls in den USA stieg nicht nur nicht an, sondern sank sogar statistisch signifikant ab (Izenman und Zabell 1981; Menaker 1970; Udry 1970). Denn ein längerer Stromausfall ist für die meisten Menschen eine belastende und beängstigende Unterbrechung ihrer Alltagsabläufe und somit gerade nicht besonders angetan für entspannte Erotik, so die unromantische Gegenthese. Ganz zu schweigen davon, dass im Stromausfall-Szenario viele Menschen gar nicht zu Hause waren, weil sie die ganze Nacht in Aufzügen und U-Bahnen feststeckten (Menaker 1970). Ähnliches trifft auf die Corona-Krise zu: Menschen in systemrelevanten Berufen sind teilweise verstärkt aushäusig unterwegs und leben an der Erschöpfungsgrenze. Und auch durch Homeoffice und Homeschooling können lusttötende Belastungen entstehen. Das romantische Bild, welches das Mehr-Beziehungssex-plus-Babyboom-Narrativ zeichnet, muss also relativiert werden.

\section{Das Erhöhte-Geburtenraten-und-erhöhte- Scheidungsraten-Narrativ}

Im Vergleich zum Stress eines stunden- oder maximal tagelangen Stromausfalls sind die wochen- und monatelangen psychosozialen, gesundheitlichen und ökonomischen Belastungen der Corona-Krise erheblich größer. So zeigen wissenschaftliche Übersichtsarbeiten, dass die psychische Gesundheit sich nach der Wirtschaftskrise im Jahr 2008 (Parmar et al. 2016) sowie im Zuge von Quarantäne-Maßnahmen (Brooks et al. 2020) signifikant verschlechtert hat. Eine Zunahme an Aggressivität, Angst, Depressivität oder gar Suizidalität wirkt sich dabei in der Regel negativ auf das Beziehungs- und Sexualleben aus. Anstelle von „Quarantäne-Honeymoon“ droht vielen Paaren vermutlich also eher eine Art „Lagerkoller“, der die Trennungsraten und amtlichen Scheidungsstatistiken hochtreiben wird, insbesondere, wenn die räumlichen Verhältnisse sehr beengt sind, psychische Erkrankungen vorliegen und bereits Paarkonflikte bestehen. Bei vermehrtem Stress in bedrohlichen Krisensituationen sind durch damit einhergehende emotionale Probleme und soziale Konflikte erhöhte Scheidungsraten und verringerte Heirats- und Geburtenraten zu erwarten (Cohan und Cole 2002).

Andererseits sagen sowohl die Bindungstheorie als auch die Terror-Management-Theorie (Rodgers et al. 2005) vorher, dass eine existenzielle Bedrohungssituation dazu führen kann, dass Menschen sich auf den Wert ihrer Beziehungen besinnen und näher zusammenrücken, somit die Scheidungsraten sinken und die Heirats- und Geburtenraten steigen. Am Beispiel der Krisensituation des Hurrican „Hugo“, der im Jahr 1989 im Südosten der USA große Schäden anrichtete und Dutzende Todesopfer forderte, wurde die Stress-These gegen die Bindungs-These getestet. Es stellte sich heraus, dass konform zur Stress-These im Nachgang der Sturm-Katastrophe die Scheidungsrate tatsächlich signifikant stieg, gleichzeitig aber gemäß Bindungs-These die Heirats- und die Geburtenrate ebenfalls stiegen (Cohan und Cole 2002; zu positiven Fertilitäts-Effekten von Naturkatastrophen und Terroranschlägen siehe zudem Evans et al. 2010; Rodgers et al. 2005).

Ein solcher gegenläufiger Trend von Stress- und Bindungs-Effekten ist auch im medialen Corona-Diskurs abgebildet. Immer wieder wurde ein Erhöhte-Geburtenraten-und-erhöhte-Scheidungsraten-Narrativ angeboten:

- Das Stadtmagazin „Tip Berlin“ zitiert den Paarberater Martin Jurock, der einerseits vermehrten Beziehungssex samt Corona-Babys und andererseits mehr Beziehungstrennungen vorhersagt.

- Der TV-Sender MDR Sachsen interviewt den Paartherapeuten und Sexualcoach Rouven Gehr, der die Corona-Krise als „Stresstest“ für Beziehungen beschreibt und eine Parallele zum Urlaub zieht, aus dem ja auch manche Paare frisch verliebt und andere mit festen Trennungsabsichten zurückkehren.

- Die „BILD“- Zeitung spitzt zu auf „mehr Liebe, mehr Hass“.

- Das Magazin „Women’s Health“ konstatiert, dass Paare jetzt zu Hause gestresst die „Wände hochlaufen“ oder auch „traute Zweisamkeit“ genießen können. Damit Letzteres klappt, werden Sex-Tipps gegeben.

Insgesamt sind beide bislang dargestellten Narrative optimistisch. Der Zugewinn an partnerschaftlicher Nähe wird begrüßt. Und der 
Glaube an den „Babyboom“, der eine positive Zukunft symbolisiert, ist ungebrochen. Der romantische Tenor der beiden Beziehungssex-Narrative kommt sprachlich auch dadurch zum Ausdruck, dass die Massenmedien hier ganz häufig von „Der Liebe in den Zeiten von Corona "schreiben, eine Anspielung auf den Roman-Bestseller „Die Liebe in den Zeiten der Cholera“ (García Márquez 1987). In der Roman-Handlung findet ein Liebespaar im ganz hohen Lebensalter endlich zusammen und nutzt die Cholera-Quarantäne auf einem Schiff für ungestörte Zweisamkeit.

Gegen die befürchtete Gefahr vermehrter Trennungen und Scheidungen bieten die in den Medien stark sichtbaren Paartherapeut:innen, Beziehungsberater:innen und Sexualcoaches Tipps und Tricks, Telefon-Sprechstunden und Online-Seminare, ermutigen Paare also zur „Arbeit an der Beziehung“. Gleichzeitig vermarkten sie ihre Profession und deren Dienstleistungen. Das Erhöhte-Geburtenraten-und-erhöhte-Scheidungsraten-Narrativ schließt mit seinen vielfältig mitgelieferten Tipps und Lösungsvorschlägen von Beziehungs- und Sex-Profis also nicht zuletzt auch an Diskurse der Selbstoptimierung, Therapeutisierung und Pädagogisierung unseres Beziehungs- und Sexuallebens an (vgl. Dinges 2017: 28).

\section{Das Weniger-unverbindlicher-Partnersex-Narrativ}

Wie die bisher zitierten Beispiele zeigen, wurden Veränderungen der Sexualität durch die Corona-Krise oft unhinterfragt am Beispiel heterosexueller monogamer Paarbeziehungen verhandelt. Ein anderes Narrativ ergibt sich, wenn man Partnersex in den Blick nimmt, der nicht mit häuslichen Ehe- und Lebenspartner:innen, sondern im Rahmen alternativer Beziehungsmodelle (z. B. Plus-Freundschaften, polyamore Beziehungsnetzwerke) stattfindet sowie als sogenannter unverbindlicher Sex (Casual Sex; Wentland und Reissing 2011) etwa im Rahmen von One-Night-Stands, Sex-Bekanntschaften, Affären, Sex-Dates oder Paysex-Kontakten praktiziert wird. Diese Formen von sexuellen Interaktionen, die Körperkontakt mit Personen außerhalb des eigenen Haushalts implizieren und unter anderem für Jugendliche, Singles und LGBTIQ-Personen häufiger zum Lebensmodell gehören, waren während der Corona-Kontaktsperren praktisch untersagt. Das bedeutet, dass viele Jugendliche und Erwachsene sich auf lange Wochen und Monate ohne intime körperliche Kontakte zu anderen Menschen einzustellen hatten.

Das entsprechende Weniger-unverbindlicher-Partnersex-Narrativ war im medialen Diskurs mit 18.7 \% prominent vertreten (siehe oben $>$ Tab. 1).

- Der Radiosender Deutsche Welle spricht von „Pause für die Libido“ und „schlechten Zeiten für Singles“.

- Das Online-Magazin „Finanznachrichten.de“ stellt fest „Deutsche bleiben enthaltsam “ und zitiert eine Umfrage unter $N=1000$ Mitgliedern der Online-Dating-Plattform Joyclub, gemäß der 38.5\% der befragten Männer und $50.5 \%$ der befragten Frauen seit Beginn der Corona-Krise weniger oder gar keinen Partnersex mehr hatten.

- Das Online-Magazin „queer.de“ berichtet, die „Mehrheit der Schwulen hat wegen Corona weniger Sex“, und bezieht sich dabei auf eine Umfrage des schwulen Dating-Portals Gay.de unter $N=1327$ Mitgliedern, der zufolge 54\% der befragten Schwulen und Bisexuellen weniger Sex hatten als vor der COVID-19-Pandemie und mehrheitlich auch keine öffentlichen Cruising-Areas mehr aufsuchten.
- Der Radiosender rbb behandelt den Sexmangel alleinlebender Erwachsener in Berlin und titelt mit dem Zitat „Sex fehlt mir tatsächlich extrem“.

Die Online-Kommentare zum letztgenannten Beitrag sind teilweise hämisch: „Leute, habt ihr keine anderen Probleme?“ und „Mein Mitleid mit diesen Personen hält sich in Grenzen“. Wer partout ein Lebensmodell jenseits der monogamen Paarbeziehung haben will, solle sich jetzt nicht über Sexmangel in der Corona-Krise beschweren, so der moralisierende Tenor der Kritiker:innen des Beitrags.

Tatsächlich tauchte das Weniger-unverbindlicher-PartnersexNarrativ in zwei Varianten auf: Einmal als deskriptives Narrativ der Beschreibung der sexuellen Verhaltensänderungen von Menschen, die sich an die Kontaktverbote hielten und dadurch ungewollten Sexmangel erlebten. Und einmal als präskriptives Narrativ, das sexuelle Verhaltensänderungen einforderte - oder gar erzwingen wollte - bei denjenigen, die möglicherweise noch außerhalb einer einzigen häuslichen Partnerschaft Sexkontakte pflegten.

Nirgends wurden die Einschränkungen beim unverbindlichen Sex so kontrovers diskutiert wie in den LGBTIQ-Szenen. Da waren diejenigen, die sich bereitwillig auf eine längere Abstinenzphase einstellten, dies in Sozialen Medien selbstgewiss verkündeten und gleich die PrEP absetzten, weil es mit dem Partyleben doch jetzt sowieso erst einmal vorbei sei. Und da waren diejenigen, die auf Sex-Dating keineswegs verzichten wollten und das auch so sagten, schließlich sei Sex für sie ein Grundbedürfnis. Sie riefen nicht nur Kopfschütteln und Wut, sondern auch Kontroll-Fantasien auf den Plan: In der neu gegründeten Facebook-Gruppe „LGBTI COVID-19 Response“ schlug ein Nutzer in einem präskriptiven Weniger-unverbindlicher-Partnersex-Narrativ beispielsweise vor, doch während der COVID-19-Pandemie am besten Grindr, Scruff, GROWLr und alle anderen queeren Dating-Apps zu schließen, um den Gelegenheitssex zu stoppen. Eine hitzige Diskussion mit Dutzenden von Beiträgen folgte.

Der Sexualpädagoge Marco Kammholz sieht im Interview mit dem Magazin „männer* “ beide Haltungen als problematisch: Das völlige Ignorieren der Corona-Gefahr und unbeirrte Fortführen diverser Sexkontakte wie bisher, aber auch den allzu bereitwilligen und moralisch überhöhten Totalverzicht auf unverbindlichen Partnersex. Einschränkungen beim Partnersex sollten stattdessen nach wohlüberlegter Risikoeinschätzung erfolgen und auch die Bedeutung von Partnersex für Wohlbefinden und Lebensfreude würdigen, so der Sexualpädagoge.

Gerade in LGBTIQ-Kontexten liegen natürlich Vergleiche zwischen Coronavirus-Narrativen und HIV-Narrativen auf der Hand. Die Etablierung von Safer Sex, TasP (Treatment as Prevention) und neuerdings PrEP (Pre-Exposure Prophylaxis) hatte im schwulen Sexualleben mit Blick auf HIV gerade zu neuer Angstfreiheit und lustfördernder Sorglosigkeit geführt (Döring 2019a). Drohen nun mit Verweis auf das viel leichter als HIV übertragbare neuartige Coronavirus neue Angst und neue Stigmatisierung nicht-monogamer Lebensweisen?

\section{Das Mehr-Telefonsex-und-mehr-Onlinesex-Narrativ}

Dass Face-to-Face-Sexkontakte mit Partner:innen außerhalb des eigenen Haushalts in Zeiten der Pandemie ein nicht unerhebliches Risiko der Transmission von SARS-CoV-2 bergen, ist angesichts seiner 
Übertragung durch Tröpfcheninfektion (also durch Husten, Niesen, Reden, Küssen) unbestritten. Was bleibt, sind aber noch die zwischenmenschlichen Sexkontakte auf Distanz. Diese sind seit den 1990er-Jahren bekannt unter Stichworten wie Cybersex, virtueller Sex, Chatsex, Camsex oder neuerdings auch Sexting (Courtice und Shaughnessy 2017; Dannecker und Lemke 2017; Döring 2000 , 2012a, 2014a; Koops et al. 2018; Shaughnessy et al. 2011). Angesichts der Corona-Kontaktverbote erfreuten sich solche medienvermittelten zwischenmenschlichen Sexkontakte plötzlich besonders großer Beliebtheit und wurden öffentlich so stark diskutiert, dass im März 2020 in den Medien ein Mehr-Telefonsex-und-mehr-Onlinesex-Narrativ als das populärste Partnersex-Narrativ (vertreten in 21.6\% der Medienbeiträge im Sample, siehe oben > Tab. 1) identifiziert werden konnte. Genau wie das Weniger-unverbindlicher-Partnersex-Narrativ existierte auch das Mehr-Telefonsex-und-mehr-Onlinesex-Narrativ in deskriptiven und präskriptiven Varianten:

- Im Interview mit dem Radiosender rbb berichtet Matthias (35) aus Berlin, dass er aktuell keine Real-Dates mehr macht. „Was ich gerade so fleißig betreibe wie nie, ist Sexting. Bilder austauschen, Dirty Talk, auch mal als Voicemail. Das tut zurzeit echt gut."

- Der Radiosender Deutschlandfunk Nova will seinem Publikum erklären, wie man trotz Kontaktsperren weiterhin Partnersex haben kann. „Safer Sexting“ und „Sex per Telefon“ sind die Empfehlungen der Sex-Bloggerin und Autorin Theresa Lachner, die der Sender weitergibt.

- Das Online-Magazin „Refinery29“ bietet einen „Sexting-Guide: Für ein aufregendes Liebesleben trotz Social Distancing“. Die Tipps stammen von der kanadischen Sexualpädagogin Samantha Bitty und umfassen Konsensfragen ebenso wie die richtige Beleuchtung und Pose für sexy Selfies und tolle Arschbilder.

- Das Magazin „Grazia“ propagiert neben „Chats, Sexting, Telefongesprächen“ auch „virtuelle Dates“ per Skype oder Facetime, zu denen man ganz stilvoll das „schönste Outfit ausführen" solle.

Das Gesundheitsamt von New York City sorgte weltweit für Schlagzeilen, als es am 21. März 2020 Tipps zum sicheren Sex während der Corona-Krise veröffentlichte und sich dabei für eine Behörde überraschend inklusiv und sexpositiv positionierte (eigene Übersetzung aus dem Englischen):

„Wenn Sie Ihre Sexualpartner normalerweise online suchen oder Ihren Lebensunterhalt mit Sex bestreiten, sollten Sie Folgendes in Betracht ziehen: Pausieren Sie mit den persönlichen Treffen. Video, Sexting oder Chat-Räume können Optionen für Sie sein.“

Bemerkenswert ist, dass der oft verpönte, gar zum pathologischen oder zumindest devianten Risikoverhalten erklärte Onlinesex einschließlich Sexting (vgl. Döring 2014a) jetzt offiziell zum Gesundheits-Präventionsverhalten avancierte: So empfahlen unter anderem die Bundeszentrale für gesundheitliche Aufklärung (BZgA) und die Aidshilfe „Online-Dates mit Video-Calls“ bzw. „Telefonsex oder Onlinesex“.

Beim Partnersex von der Face-to-Face-Variante auf medienvermittelte Formen umzusteigen, um eine SARS-CoV-2-Übertragung zu vermeiden, wurde nicht nur bei privaten, sondern auch bei kom- merziellen Partnersex-Interaktionen beobachtet und empfohlen. Der Berufsverband erotische und sexuelle Dienstleistungen BesD e.V. riet Sexdienstleistenden von persönlichen Treffen mit Kund:innen während der COVID-19-Pandemie ab und benannte alternative Verdienstmöglichkeiten:

„Innerhalb der Branche bieten sich Webcamming, Phone und Direct Chats sowie der Verkauf von Adult Content (Fotos, Videos) auf Plattformen an, wo kein Körperkontakt stattfindet. Eine Auswahl von Plattformen sind zum Beispiel adultwork, streamate, Big7, my dirty hobby, onlymevip oder chaturbate, sexpanther und adulttime."

Im Rahmen des Mehr-Telefonsex-und-mehr-Onlinesex-Narrativs berichten Medien über den großen Ansturm auf Online-DatingApps und Sexkontakt-Portale. Konkrete Online-Plattformen und Apps werden dabei angeführt (z. B. Tinder, Parship, Bumble, TheCasualLounge.ch) und Corona-Spezialangebote ausführlich gewürdigt (z. B. die kostenlose Freischaltung der Tinder-Funktion „Passport“ für weltweite Kontaktsuche bis zum 30. April 2020). Der Tenor der Medienbeiträge mit dem Mehr-Telefonsex-und-mehr-OnlinesexNarrativ ist positiv bis enthusiastisch. Das ist gute PR für die Online-Dating-Branche. Denn ihr gelingt es, mit ihren Corona-Spezialangeboten sowie mit Ergebnissen Corona-bezogener Mitglieder-Befragungen immer wieder in den Medien erwähnt zu werden. Als Service-Leistungen werden in den Medien zusammen mit dem Mehr-Telefonsex-und-mehr-Onlinesex-Narrativ oftmals Tipps und Tricks weitergegeben. Derartige Hinweise sind durchaus sinnvoll, da Telefon- und Onlinesex neben dem technischen Equipment spezifische Kenntnisse und Fähigkeiten erfordern.

Viele Tipps in den Mainstream-Medien sind dabei auf OnlinesexInteraktionen zwischen zwei Personen bzw. zwischen „Mann und Frau“ bezogen. Spezifische Fragen, die sich z. B. auf die medienvermittelte Umsetzung von queeren, Gruppensex-, BDSM- oder Fetisch-Interaktionen beziehen, werden in den Online-Communitys der jeweiligen Szenen verhandelt. So wurde beispielsweise in der Facebook-Gruppe „LGBTI COVID-19 Response“ die folgende Anfrage gestellt (eigene Übersetzung aus dem Englischen):

\begin{abstract}
„Meine Freunde sprechen über die Möglichkeiten, online sexuell zu spielen - Telefonsex, Camsex, Sex-Apps - aber sie wissen nicht, wie sie es machen sollen. Vorschläge für Optionen, die besten Apps, wie man seine Privatsphäre schützen kann, wie man verhindert, dass von der Online-Aktion heimlich Aufzeichnungen gemacht werden usw.? Und vor allem: der beste Einsatz von Kamera und Beleuchtung..."
\end{abstract}

Die Antworten aus der Community umfassten Tipps für Plattformen und Apps (z. B. Spicer, Houseparty, Snapchat, Zoom, Kik, Omegle, Grindr) sowie auch für Sextoys, die über Entfernung gesteuert werden können (z. B. Produkte von We-Vibe), für bestimmte Medienformen (z. B. selbstkreierte erotische Audioaufnahmen) sowie für neue Angebote virtueller queerer Sex-Partys: Verwiesen wurde z. B. auf die virtuelle Version von „Inferno NYC“, auf die virtuelle „Lock Down Disco“ mit Instagram-DJs, Chat-Room und Skype-Darkroom und nicht zuletzt auf die Online-Ausgabe von Londons „Naked Gay Party SBN“, die offline normalerweise rund 600 
Gäste zählt, am 29. März 2020 aber mehr als 3000 Teilnehmer im Video-Chat begrüßte.

Auch wenn deutliche Zuwächse bei der Nutzung von Datingund Sex-Apps zu erwarten sind, sollte der im Narrativ propagierte Onlinesex-Boom nicht überschätzt werden. Denn die Zuwendung zu Dating-Apps und Sexkontakt-Portalen erfolgt häufig auch aus nicht-sexuellen Motiven: Es geht um Ablenkung, Zeitvertreib, Neugier und Geselligkeit (Timmermanns und De Caluwé 2017). So mag in Corona-Zeiten auf Sexkontakt-Plattformen eben manchmal gar kein schneller Onlinesex gesucht werden, sondern eher Unterhaltung und Sozialkontakt.

Bei der kritischen Einordnung des Mehr-Telefonsex-und-mehrOnlinesex-Narrativs während der COVID-19-Pandemie ist nicht zuletzt dessen affirmative Grundhaltung zu hinterfragen. So werden mögliche Probleme und Risiken, die bislang den Cybersex- und Sexting-Diskurs womöglich übermäßig stark geprägt haben, nun plötzlich gar nicht mehr erwähnt: Sexuelle Online-Belästigung, Rache-Pornografie, Online-Stalking, Datenschutzprobleme, betrügerische Online-Angebote usw. Dieser Umschwung in der Bewertung ist zwiespältig: Einerseits ist die Entstigmatisierung und Normalisierung mediatisierter sexueller Interaktionen zu begrüßen, da diese ja ohnehin seit mittlerweile rund einem Vierteljahrhundert zum sexuellen Repertoire unterschiedlicher Bevölkerungsgruppen gehören und mehrheitlich positiv erlebt werden (Döring und Mohseni 2018). Andererseits ist es problematisch, wenn die Würdigung der Qualitäten von medienvermitteltem Partnersex - speziell seiner Nützlichkeit während Face-to-Face-Kontaktsperren - gleich in Glorifizierung und regelrechte Werbung übergeht.

\section{Solosex-Narrative}

Neben den vier Corona-bezogenen Partnersex-Narrativen konnten auch vier Solosex-Narrative identifiziert werden. Mit Solosex ist Masturbation bzw. Selbstbefriedigung gemeint.

\section{Das Mehr-Masturbation-Narrativ}

Nachdem unter anderem Medizin und Pädagogik die Masturbation jahrhundertelang wegen ihrer angeblichen Schädlichkeit bekämpft hatten, wird sie inzwischen evidenzbasiert als förderlich für die psychische und physische Gesundheit eingestuft (Coleman 2003; Levin 2007; Robbins et al. 2011). Stärkung des Immunsystems, Abbau von Stress, Ablenkung bei Langeweile und Ersatz für Partnersex - in der Corona-Isolation waren das plötzlich hochgradig wünschenswerte Eigenschaften der Selbstliebe. In den Medien war dementsprechend ein deskriptives Mehr-Masturbation-Narrativ zu finden, das die verstärkte Zuwendung zur Selbstbefriedigung beschrieb, aber auch ein präskriptives Mehr-Masturbation-Narrativ, das mehr Masturbation ausdrücklich empfahl:

- „Weniger Hochzeitspläne, mehr Masturbation“ titelt die österreichische Tageszeitung „Kurier“ und beruft sich auf eine Umfrage des Sextoy-Herstellers We-Vibe unter $N=1200$ liierten Menschen, der gemäß 54\% der Paare in gemeinsamer Quarantäne und 73\% der getrennt lebenden Paare in der CoronaKrise mehr Lust auf Solosex verspüren.

- Die „BILD“-Zeitung macht auf mit „Selbstbefriedigung gegen Corona: Warum Orgasmen jetzt besonders wichtig sind“ und führt Stressreduktion und Stärkung des Immunsystems an.
- „Der sicherste Sexpartner ist man selbst“, zitiert die Tageszeitung „taz“ die Empfehlung des New Yorker Gesundheitsamtes, persönliche Sextreffen in der Corona-Krise entweder durch Onlinesex (siehe oben: Mehr-Telefonsex-und-mehr-Onlinesex-Narrativ) oder eben durch Masturbation zu ersetzen.

Das präskriptive Mehr-Masturbation-Narrativ findet sich in der Presse und in den Veröffentlichungen diverser Gesundheitsbehörden, nicht nur des New Yorker Gesundheitsamtes. So erklärt die BZgAKampagne „Liebesleben“:

„Gerade für Singles oder Menschen mit wechselnden (Sex-) Partnerinnen und (Sex-)Partnern bedeutet Corona, dass man Sex mit anderen Menschen, wie auch den generellen Kontakt, zwar vermeiden sollte - man kann sich aber auch auf sich und seine eigene Sexualität fokussieren. Denn auch ,Sex mit sich selbst‘ kann spannend, lustvoll und reizvoll sein. Du kannst deinen Körper entdecken und herausfinden, was dir wie am bestens gefällt, ob du beispielsweise Sextoys magst oder nicht. Und es besteht bei Selbstbefriedigung kein Risiko für eine Infektion mit dem neuartigen Coronavirus!“

Der neue Enthusiasmus, mit dem Medien und Gesundheitsbehörden die Masturbation feiern, mag ein wünschenswerter Schritt zu weiterer Enttabuisierung und Normalisierung dieser sexuellen Ausdrucksform sein. Gleichzeitig ist es problematisch, wenn Selbstbefriedigung als Alternative zum Partnersex empfohlen wird. Denn als dauerhafter Ersatz für zwischenmenschliche Intimität kann sie kaum dienen. Der sexpositive Tenor des Mehr-Masturbation-Narrativs droht dort in Repression umzuschlagen, wo damit gleichzeitig ganz selbstverständlich der Totalverzicht auf unverbindlichen Partnersex gefordert wird.

\section{Das Mehr-Sextoys-Narrativ}

Das während der COVID-19-Pandemie populärste Solosex-Narrativ (14.4\%, siehe oben $>$ Tab. 1 ) postuliert die Verwendung von mehr Sexspielzeug. Toys kommen beim Partnersex, noch häufiger - vor allem bei Frauen - aber beim Solosex zum Einsatz (Döring und Poeschl 2019), für den während der Corona-Isolation laut gängiger Annahme besonders viel Gelegenheit bestand:

- „Wo es viele Corona-Fälle gibt, steigt der Verkauf von Sextoys“ erklärt die Tageszeitung „Die Welt“. Im Beitrag werden für Deutschland 20- bis 30-prozentige Umsatz-Zuwächse während der COVID-19-Pandemie bei Unternehmen wie We-Vibe und Amorelie.de berichtet, wobei der „Womanizer“-Vibrator und „Vibro-Eier“ nicht nur als Verkaufsschlager vorgestellt werden, sondern die entsprechenden Online-Shopping-Seiten direkt verlinkt sind.

- „Viel Corona = Viele Sextoys“ stellt der private Rundfunksender RTL fest und verweist wiederum auf die Zuwächse bei Marken wie We-Vibe.com und Womanizer.com

- „Schweizer in Quarantäne stürmen Sextoy-Shops“ weiß das Online-Magazin „Nau“ und nennt zudem 160-prozentige Zuwächse beim Verkauf von Dildos beim Schweizer Online-Versandhändler Galaxus.ch. 
Das Mehr-Sextoys-Narrativ ist optimistisch ausgerichtet und verweist auf besonders lustvolle Selbstbefriedigung dank mehr oder minder raffinierter technischer Hilfsmittel. Solche massenmedialen Informationen über Sexspielzeuge haben normalisierenden und aufklärenden Charakter. Gleichzeitig handelt es sich um geschickte Öffentlichkeitsarbeit der Sextoy-Branche, denn konkrete Marken und Produkte wurden in den Medienbeiträgen oft lobend genannt und teilweise auch direkt verlinkt.

Was in diesem Narrativ fehlt, ist eine kritische Auseinandersetzung mit den Produkten, etwa ihrer Funktionalität, Nachhaltigkeit, Materialqualität oder Preisgestaltung. Während es zahlreiche Weblogs gibt, die sich der differenzierten Rezension von Sextoys aus Nutzer:innensicht widmen (Döring 2019b), kommen in den Medienbeiträgen immer nur die Marketing-Chef:innen der Firmen zu Wort, die über Verkaufserfolge berichten. Nicht zuletzt ist anzumerken, dass sich das Mehr-Sextoys-Narrativ auf etablierte Toys wie Dildos und Vibratoren bezieht und Sexpuppen oder Sexroboter, die anderweitig als neue Trends diskutiert werden (Döring 2017; Döring und Poeschl 2018), ausblendet. Dabei sind Ganzkörper-Toys wie Roboter und Puppen, aber auch herkömmliche Kuscheltiere womöglich besonders gefragt, wenn es in der Corona-Isolation um Bedürfnisse nach Zärtlichkeit, Umarmung und Kuscheln geht und man sich etwa beim Einschlafen beruhigen und trösten möchte.

\section{Das Mehr-Pornografie-Narrativ}

Bei der Selbstbefriedigung wird nicht nur zu Toys gegriffen, sondern oft auch zur Pornografie, meist in Form von Online-Pornografie (Döring 2019c). Während der COVID-19-Pandemie war das verstärkt der Fall - so behauptet es zumindest das in den Medien verbreitete Mehr-Pornografie-Narrativ: „Corona lässt Pornokäufe explodieren“ titelte bei weitem nicht nur die „BILD“-Zeitung:

Einen besonderen „PR-Coup“ landete Pornhub.com. Dieser führende Anbieter von Online-Pornografie sorgte Anfang März 2020 weltweit für Schlagzeilen, weil er den in Quarantäne lebenden Italiener:innen einen Monat Premium-Zugang auf die Plattform schenkte. Das Angebot wurde so stark befürwortet und nachgefragt, dass Pornhub es sofort auf Spanien und Frankreich und schließlich auf die ganze Welt erweiterte. Am 24. März 2020 twitterte Pornhub (@pornhub) (eigene Übersetzung aus dem Englischen):

„Bleiben Sie zu Hause und helfen Sie mit, die Infektions-Kurve abzuflachen! Da COVID-19 weiterhin Auswirkungen auf uns alle hat, hat Pornhub beschlossen, den kostenlosen PornhubPremiumzugang weltweit bis zum 23. April zu verlängern. Genießen Sie also, bleiben Sie zu Hause und bleiben Sie sicher.“

Wer sich kostenlos anmeldete, bestätigte gleichzeitig, sich von Sozialkontakten fernzuhalten und dafür Pornhub-Premium-Videos zu genießen. Pornhub benannte sich selbst scherzhaft in „Stayhomehub“ um. Für die im Mehr-Pornografie-Narrativ immer wieder genannte Plattform Pornhub dürfte sich im Zuge der Corona-Krise ein deutlicher Bekanntheits- und Image-Gewinn ergeben haben. Dabei war der Ansatz von Pornhub keineswegs originell. Diverse Unternehmen boten während der Corona-Krise kostenlose Premiumzugänge an. Im Zusammenhang mit Sex bzw. Porno hatte diese Marketingmaßnahme jedoch einen besonders hohen Nachrichtenwert.
Im Vergleich mit den sonst äußerst polarisierten öffentlichen und akademischen Debatten über Pornografie wirken die Medienbeiträge mit dem sowohl deskriptiv als auch präskriptiv ausgerichteten Mehr-Pornografie-Narrativ überraschend entdramatisiert. Statt sich über die sonst recht alarmistisch diskutierten möglichen Pornografie-Risiken wie Sucht, Selbstwertverunsicherung, sexuelle Unzufriedenheit, Erektionsprobleme, Sexismus, Verrohung und Gewalt (vgl. Döring 2012b; Lemke und Weber 2016) zu sorgen, ist man während der Corona-Krise offenbar vor allem froh, wenn die Menschen dank Pornokonsum zu Hause bleiben und die Kontaktsperren einhalten. Hier findet also eine prinzipiell wünschenswerte Entstigmatisierung der Nutzung von Erotika und Pornografien statt, für die die Forschung ja neuerdings tatsächlich verstärkt auch Positiveffekte nachweist (Döring 2019c). Dass damit aber gleich jegliche kritische Auseinandersetzung mit Pornografienutzung entfällt, ist bedauerlich. In seiner unkritisch-affirmativen und kommerzorientierten Ausrichtung besteht zwischen dem Mehr-PornografieNarrativ und dem Mehr-Sextoys-Narrativ sowie dem Mehr-Telefonsex-und-mehr-Onlinesex-Narrativ jeweils eine deutliche Parallele.

\section{Das Neues-Genre-Coronavirus-Pornos-Narrativ}

Die mediale Öffentlichkeit bewegte nicht nur, dass Menschen während der Corona-Krise mehr Pornografie rezipierten, sondern auch, dass sie sich plötzlich einer neuen Pornografie-Gattung zuwandten, nämlich dem Genre der Coronavirus-Pornos. „Sex-Trend extrem die Zahl der Coronavirus-Pornos steigt rasant an“ meldete das Online-Magazin „Tonight News“.

In den sogenannten Coronavirus-Pornos spielt die Handlung im Krankenhaus oder vermeintlich in der chinesischen Stadt Wuhan, in der COVID-19 ursprünglich ausbrach. Die Protagonist:innen tragen Atemschutzmasken, Handschuhe und Schutzanzüge, wie unter anderem das Magazin „Vice“ detailliert beschreibt. Tatsächlich fanden sich auf der Pornografie-Plattform Pornhub.com Anfang April unter dem Suchbegriff „Corona“ mehr als 1000 Videos, auf der Plattform XHamster.com deutlich mehr als 100.

Was hier zum angeblich extremen, zumindest aber merkwürdigen „Sex-Trend“ erklärt wurde, ist dabei eigentlich ganz normal: Aktuelle Ereignisse spiegeln sich in den sexuellen Fantasien der Menschen wider und werden somit auch zum Thema der Pornografie. Dabei können Coronavirus-Fantasien und Coronavirus-Pornos vermutlich ganz unterschiedliche Funktionen erfüllen: Angstabwehr, Erotisierung des Bedrohlichen, Neugier auf das Skurrile, Lust an der Grenzüberschreitung, Genesungshoffnungen usw. Zu beachten ist auch, dass der Coronavirus-Topos an etablierte Kinkund Fetisch-Motive (etwa Kliniksex, Latexmasken und -anzüge) anschließt. Die vom Anbieter Pornhub.com veröffentlichten Plattform-Statistiken zur COVID-19-Pandemie belegen ein im März 2020 deutlich gestiegenes Interesse an Coronavirus-Pornos. Diese Statistiken wurden in den entsprechenden Medienbeiträgen oft zitiert, was wiederum der Bekanntheit der Marke Pornhub zugutegekommen sein dürfte.

\section{Sexualbezogene Problem-Narrative}

Abzuheben von den überwiegend affirmativ-optimistischen Partnersex- und Solosex-Narrativen sind politisch ausgerichtete Narrative, die sich auf Probleme beziehen, sexuelle und reproduktive 
Gesundheit und Rechte während der Corona-Krise zu sichern und zu wahren, insbesondere bei vulnerablen Gruppen. Die ProblemNarrative sind in der Mehrzahl der Medienbeiträge zu finden (siehe oben $>$ Tab. 1).

\section{Das Mehr-sexualisierte-häusliche-Gewalt-Narrativ}

Dass es in häuslicher Corona-Isolation zu einer Gewalteskalation kommt und Betroffene noch schwerer als sonst Hilfe suchen und erhalten können, ist die Prognose des Mehr-sexualisierte-häuslicheGewalt-Narrativs. „Man muss mit dem Schlimmsten rechnen“ ist dabei eine mehrfach, unter anderem auch von der „Tagesschau“, benutzte Formulierung. Neben Warnungen vor der befürchteten Gewalteskalation werden - entsprechend den Kriterien einer qualitätsvollen Berichterstattung über sexuelle Gewalt gegen Kinder (Döring und Walter 2019) - auch Lösungsmöglichkeiten aufgezeigt: Medienbeiträge publizieren viel häufiger als sonst TelefonHotlines und Online-Anlaufstellen. Auch berichten sie von den Forderungen der Frauenhaus-Vertreterinnen, die Plätze müssten jetzt dringend aufgestockt werden, um der vermutlich steigenden Nachfrage zu entsprechen.

\section{Das Mehr-Zugangshürden-zum-Schwangerschafts- abbruch-Narrativ}

Das Mehr-Zugangshürden-zum-Schwangerschaftsabbruch-Narrativ weist darauf hin, dass die notwendigen Termine für einen Schwangerschaftsabbruch während der Corona-Maßnahmen nicht fristgerecht erledigt werden können und somit die Gefahr besteht, dass es bei ungewollt Schwangeren zu Suiziden und unprofessionellen Abtreibungen kommt. Die Tageszeitung „taz“ unterstrich dies am 23. März 2020 mit dem Symbolbild eines Drahtbügels unter dem Titel „Blutungen bis zum Tod“. Schon drei Tage später meldete „Die ZEIT“, dass sich die Familienministerin mit den Ländern darauf geeignet habe, in Deutschland während der COVID-19-Pandemie die Schwangerschaftskonfliktberatung auch telefonisch oder online durchzuführen. Die Forderung von Pro-Choice-Aktivistinnen, zum Abbau der Zugangshürden einen telemedizinisch begleiteten medikamentösen Abbruch zu Hause zu ermöglichen, wie er in anderen Ländern bereits Praxis und auch wissenschaftlich positiv evaluiert ist (Raymond et al. 2019), wurde in den Medien zwar vereinzelt aufgegriffen, politisch aber nicht erfüllt.

\section{Das Mehr-Ungewissheiten-bei-Schwangerschaft- und-Geburt-Narrativ}

Die existenziellen Erfahrungen rund um Schwangerschaft und Geburt gehen generell oft mit Ängsten und Unsicherheiten einher. Unter den Bedingungen einer Pandemie sind diese noch intensiviert: Ansteckungsrisiken sind ein Thema, Versorgungsengpässe in Kliniken und auch die Frage, ob trotz Kontaktsperren eine persönliche Begleitperson mit in den Kreißsaal genommen werden darf. Die massenmedialen Berichte griffen solche Sorgen und Ängste auf: „Die Unsicherheit bei Schwangeren wächst“ titelt „Die Süddeutsche“ und die „Hessenschau“ berichtet: „Schwangere verzweifeln in Corona-Krise: Keine Papas mehr im Kreißsaal“. Andere Medienberichte, etwa im Magazin ,jetzt.de“, liefern Lösungsansätze und verweisen auf virtuelle Hebammenbegleitung, Facebook-Gruppen für Schwangere und Online-Geburtsvorbereitungskurse.
Bianca Claßen ist mit dem Kanal „Bibis Beautypalace“ seit Jahren Deutschlands erfolgreichste YouTuberin (Döring 2019d). Am 20. März 2020 hat sie ihr zweites Kind entbunden und wurde im Kreißsaal in sehr angespannter Stimmung begrüßt mit den Worten „Gucken Sie, dass Sie so schnell, wie Sie können, entlassen werden! Das hier ist grade wie eine Weltwirtschaftskrise und ein dritter Weltkrieg.“ - so erzählt sie es im Video „Geburtsbericht - Unsere Tochter Emily ist da“ (Minute 3:26). Mehr als zwei Millionen Aufrufe verzeichnet das Video, in dem die Influencerin und ihr Mann von ihren eigenen Unsicherheiten berichten, letztlich aber Corona-Maßnahmen auf Entbindungsstationen als sinnvoll anerkennen.

\section{Mehr-existenzbedrohliche-Lagen-in-der-Sexarbeit- Narrativ}

Fast jeder fünfte Medienbeitrag im Sample (18.4\%, siehe oben - Tab. 1) berichtet über die existenzbedrohlichen Lagen für Prostituierte, die sich aus Bordellschließungen und Verboten körpernaher Dienstleistungen ergeben und wegen der meist fehlenden Rücklagen zu akuter Not und Obdachlosigkeit führen. „Prostituierte zwischen Telefonsex und Hartz IV. Coronavirus bedroht Existenz von Sexarbeitern “ titelt entsprechend das SWR-Fernsehen.

Interessanterweise sprechen die Medienbeiträge mit dem Mehrexistenzbedrohliche-Lagen-in-der-Sexarbeit-Narrativ nicht nur „über“ Sexarbeiter:innen, sondern tatsächlich mit ihnen. So kommen Fachberatungsstellen, der Berufsverband erotische und sexuelle Dienstleistungen BesD e.V. und einzelne Sexarbeiterinnen immer wieder ausführlich zu Wort. Angesichts der polarisierten Debatten zur Prostitution, die nicht selten ohne die Betroffenen geführt werden und das Anbieten und/oder Nachfragen von sexuellen Dienstleistungen moralisch verurteilen (vgl. Döring 2014b, 2018), ist es bemerkenswert, dass im medialen Corona-Sex-Diskurs die Interessen und Arbeitsrechte der Sexarbeitenden so gut sichtbar auf die Agenda gelangt sind. Repressive Regelungen im neuen Prostituiertenschutzgesetz, z. B. dass Prostituierte nicht in Bordellen übernachten dürfen, wurden während der Krisenzeit teilweise aufgehoben. Viele Probleme aber bleiben bestehen, und die Medien berichten über Forderungen nach staatlichen Hilfen sowie über Gutschein- und Spenden-Aktionen. Neben Prostituierten können auch Pornodarstellende während der Pandemie kaum arbeiten, es sei denn, sie stellen auf Solo-Online-Dienstleistungen um. Kleine Sexshops ohne Online-Versand sind laut Medienberichten ebenfalls in ihrer Existenz bedroht.

\section{Das Mehr-Diskriminierung-von-LGBTIQ-Personen- Narrativ}

Der Lesben- und Schwulenverband LSVD hat eine Informationssammlung zu Auswirkungen von COVID-19 auf das Leben von LGBTIQ-Personen zusammengestellt, die zahlreiche Problembereiche anspricht und auf Hilfsangebote verweist. So wird beispielsweise auf die Situation von Trans*-Personen in der Transition, von bi-nationalen Regenbogenfamilien oder geflüchteten LGBTIQ-Personen verwiesen. Die meisten dieser Probleme tauchten im Untersuchungszeitraum nicht im medialen Diskurs auf. Wenn Benachteiligungen von LGBTIQ-Personen während der COVID-19-Pandemie besprochen werden, dann konzentriert sich das Mehr-Diskriminierung-von-LGBTIQ-Personen-Narrativ im Frühjahr 2020 vor allem darauf, dass religiöse „Hassprediger“ COVID-19 als „Strafe Gottes“ 
für Homo- oder Transsexualität einordnen und das neuartige Coronavirus als „Homovirus“ bezeichnen, wie z. B. „queer.de“ und „schwulissimo.de“ berichten.

\section{Diskussion}

Bei der vorliegenden Studie handelt es sich um die erste empirische Untersuchung medialer Narrative zu sexualitätsbezogenen Auswirkungen der COVID-19-Pandemie. Sie zeigt, dass während der Pandemie vielfältige Aspekte von Sexualität medial verhandelt wurden. Im Fokus standen dabei oft Probleme wie sexualisierte Gewalt, Zugang zum Schwangerschaftsabbruch oder Bedingungen der Sexarbeit, die scheinbar häufiger als sonst unter aktiver Einbeziehung der Betroffenen thematisiert wurden, auf Hilfsmöglichkeiten verwiesen und politische Verbesserungsmaßnahmen forderten. Andererseits wurden auch sexuelle Chancen der Pandemie diskutiert, etwa mehr Partnersex oder mehr und besserer Solosex dank Toys und Pornos. Hier war die Auseinandersetzung anscheinend sexpositiver, kommerzfreundlicher und unkritischer als üblich.

In den Medien sind eindeutig negative oder positive Meta-Narrative sehr viel verbreiteter als ambivalente, obwohl die Annahme zwiespältiger Corona-Effekte sehr plausibel ist. So stand in den Medien beispielweise dem Mehr-sexualisierte-häusliche-Gewalt-Narrativ keinerlei Diskussion zu sexueller Gewalt in anderen Kontexten (z. B. Schule, Arbeitsplatz, Verein, öffentlicher Raum) gegenüber, wo sie während der Corona-Maßnahmen möglicherweise deutlich rückläufig war. Können im Zuge von Corona-Kontaktsperren vielleicht bestimmte Missbrauchs- und Ausbeutungsbeziehungen beendet werden, wenn die Betroffenen genau in dieser Zeit gezielte Hilfen bekommen? Da das eindeutig negative Narrativ zunehmender häuslicher Gewalt die öffentliche Debatte so stark dominierte, blieb eine Debatte anderer Gewaltkontexte und eventuell rückläufiger Entwicklungen bei sexuellen Übergriffen völlig aus.

Ebenso fehlte im Frühjahr 2020 eine Diskussion oder Spekulation über mögliche langfristige Folgen. Im Gewaltkontext könnte man sich beispielsweise fragen, ob im Nachgang der Corona-Pandemie möglicherweise mehr soziale Distanz im Alltag zur Norm wird und damit auch bestimmte sexualisierte Übergriffe, die sich zuvor leichter als harmloser oder gar freundlicher Körperkontakt tarnen konnten, zurückgehen werden. Gleichzeitig lässt sich fragen, ob dafür dann andere Formen von Übergriffen (z. B. OnlineBelästigung) zunehmen.

Es ist bislang noch weitgehend unklar, inwiefern die medialen Corona-Sex-Narrative tatsächliche sexualbezogene Veränderungen im Leben der Bevölkerung umfassend und akkurat abbilden, da dazu empirische Daten aus wissenschaftlichen Studien fehlen. Erste Befragungsstudien gingen zum Untersuchungszeitpunkt gerade erst ins Feld, etwa eine vom Kinsey-Institut durchgeführte dreiwellige Längsschnittstudie zu Sexualität und Beziehungen in Zeiten von Corona, eine mehrsprachige Umfrage zur Trans*Versorgung während der COVID-19-Pandemie von einem Netzwerk von Trans* Organisationen und Forschungseinrichtungen sowie eine vom Institut für Sexualpädagogik isp initiierte Studie zu Situation und Sichtweisen von Fachkräften der Sexuellen Bildung in der Corona-Krise. Die vorliegende Medieninhaltsanalyse unterliegt einer Reihe von Limitationen. So ist das Sample auf nicht-fiktionale massenmediale Online-Beiträge aus dem Februar und März 2020 beschränkt. Wei- tere Materialkorpora sollten untersucht werden (z. B. YouTube-Videos). Besonders spannend wäre zudem eine Längsschnittanalyse der Mediendarstellungen im Zeitverlauf der Pandemie. Sollte die COVID-19-Pandemie auch in fiktionalen Medienformaten wie Fernseh-Serien oder Reality-TV-Formaten aufgegriffen werden, könnte an die Forschung zu Sexualitätsdarstellungen in diesen Formaten angeschlossen werden (vgl. Lemke und Tornow 2018a, 2018b).

Medieninhaltsanalysen verfolgen das primäre Ziel, die mediale Wirklichkeit zu rekonstruieren und oft auch mit der empirischen Wirklichkeit zu konfrontieren. Darüber hinaus lässt die Analyse der Medieninhalte interpretative Rückschlüsse zu auf Fragen der Produktion (z. B. Wie recherchieren Journalist:innen das Thema „Sex und Corona“?) sowie der Rezeption und Wirkung (z. B. Für welche Corona-Sex-Narrative interessiert sich das Publikum besonders? Inwiefern lässt sich das Publikum von medialen Corona-Sex-Narrativen über Onlinesex, Sextoys oder Pornografie in seinen Einstellungen und Verhaltensweisen beeinflussen?). Solche Rückschlüsse müssen dann aber durch Befragungen der Produzent:innen und Rezpient:innen der entsprechenden Medienbeiträge geprüft werden.

Interessenkonflikt

Die Autor_innen geben an, dass kein Interessenkonflikt besteht.

\section{Literatur}

Alpalhão M, Filipe P. (2020). The Impacts of Isolation Measures against SARS-CoV-2 Infection on Sexual Health. AIDS Behav 2020; doi:10.1007/s10461-020-02853-x

Brooks SK, Webster RK, Smith LE, Woodland L, Wessely S, Greenberg N, Rubin G]. The Psychological Impact of Quarantine and How to Reduce It: Rapid Review of the Evidence. Lancet 2020; 395: 912-920

Brunvand JH. The Baby Train and Other Lusty Urban Legends. New York, NY: W.W. Norton 1993

Cohan CL, Cole SW. Life Course Transitions and Natural Disaster: Marriage, Birth, and Divorce Following Hurricane Hugo. J Fam Psychol 2002; 16 : 14-25

Coleman E. Masturbation as a Means of Achieving Sexual Health. J Psychol Human Sex 2003; 14: 5-16

Courtice EL, Shaughnessy K. Technology-Mediated Sexual Interaction and Relationships: A Systematic Review of the Literature. Sex Relation Ther 2017; 32: 269-290

Dannecker M, Lemke R. Die Lust im und mit dem Netz. Schwule Männer auf Chat- und Datingportalen. In: Dannecker M, Hrsg. Faszinosum Sexualität. Gießen: Psychosozial 2017; 173-196

Dinges M. Sexualität in Deutschland (1933-2016). In: Stiftung Männergesundheit, Hrsg. Dritter Deutscher Männergesundheitsbericht. Gießen: Psychosozial 2017; 23-38

Döring N. Feminist Views of Cybersex: Victimization, Liberation, and Empowerment. Cyberpsychol Behav 2000; 3: 863-884

Döring N. Erotischer Fotoaustausch unter Jugendlichen: Verbreitung, Funktionen und Folgen des Sexting. Z Sexualforsch 2012a; 25: 4-25

Döring N. Sexuell explizite Inhalte in neuen Medien: Negative und positive Wirkungen auf unterschiedliche Bevölkerungsgruppen. In: Reinecke L, Trepte S, Hrsg. Unterhaltung in neuen Medien. Perspektiven zur 
Rezeption und Wirkung von Online-Medien und interaktiven Unterhaltungsformaten. Köln: Herbert von Halem 2012b; 361-378

Döring N. Consensual Sexting among Adolescents: Risk Prevention through Abstinence Education or Safer Sexting? Cyberpsychology 2014a; 8: 9

Döring N. Prostitution in Deutschland: Eckdaten und Veränderungen durch das Internet. Z Sexualforsch 2014b; 27: 99-137

Döring N. Medien und Sexualität. In: von Gross F, Meister DM, Sander U, Hrsg. Medienpädagogik - ein Überblick. Weinheim: Beltz Juventa 2015; 323-364

Döring N. Vom Internetsex zum Robotersex: Forschungsstand und Herausforderungen für die Sexualwissenschaft. Z Sexualforsch 2017; 30 35-57

Döring N. Das neue Prostituiertenschutzgesetz: Wie ist es aus fachlichen Perspektiven zu beurteilen? Eine Einführung. Z Sexualforsch 2018; 31: 44-56

Döring N. Medikamentöser HIV-Schutz mittels PrEP: Aktueller Diskussionsund Forschungsstand. Z Sexualforsch 2019a; 32: 189-197

Döring N. Sexualbezogene Online-Fortbildung für Fachkräfte: Weblogs. Z Sexualforsch 2019b; 32: 100-106

Döring N. Sexuelle Aktivitäten im digitalen Kontext. Psychotherapeut 2019c; 64: 374-384

Döring N. Videoproduktion auf YouTube: Die Bedeutung von Geschlechterbildern. In: Dorer J, Geiger B, Hipfl B, Ratković V, Hrsg. Handbuch Medien und Geschlecht. Perspektiven und Befunde der feministischen Kommunikations- und Medienforschung. Wiesbaden: Springer Fachmedien 2019d; 1-11

Döring N, Bortz J. Forschungsmethoden und Evaluation in den Sozial- und Humanwissenschaften. Springer-Lehrbuch. 5. Auflage. Berlin, Heidelberg: Springer 2016

Döring N, Mohseni MR. Are Online Sexual Activities and Sexting Good for Adults' Sexual Well-Being? Results from a National Online Survey. Int J Sex Health 2018; 30: 250-263

Döring N, Poeschl S. Sex Toys, Sex, Dolls, Sex Robots - Our Under-Researched Bed-Fellows. Sexologies 2018; 27: e51-e55

Döring N, Poeschl S. Experiences With Diverse Sex Toys among German Heterosexual Adults: Findings From a National Online Survey. J Sex Res 2019; doi: 10.1080/00224499.2019.1578329

Döring N, Walter R. Media Coverage of Child Sexual Abuse: A Framework of Issue-Specific Quality Criteria. J Child Sex Abus 2019; doi: 10.1080/10538712.2019.1675841

Evans RW, Hu Y, Zhao Z. The Fertility Effect of Catastrophe: US Hurricane Births. J Popul Econ 2010; 23: 1-36

García Márquez G. Die Liebe in den Zeiten der Cholera. Köln: Kiepenheuer \& Witsch 1987

Hall KS, Samari G, Garbers S, Casey SE, Diallo DD, Orcutt M, Moresky RT, Martinez ME, McGovern T. Centring Sexual and Reproductive Health and Justice in the Global COVID-19 Response. Lancet 2020, 395: $1175-1177$.

Hussein J. Covid-19: What Implications for Sexual and Reproductive Health and Rights Globally? Sex Reprod Health Matters 2020; 28: 1746065

Izenman AJ, Zabell SL. Babies and the Blackout: The Genesis of a Misconception. Soc Sci Res 1981; 10: 282-299

Koops T, Dekker A, Briken P. Online Sexual Activity Involving Webcams - An Overview of Existing Literature and Implications for Sexual Boundary Violations of Children and Adolescents. Behav Sci Law 2018; 36: 182-197

Lemke R, Tornow T. Darstellungen von Sexualität in den Massenmedien: Ansätze, Theorien und Befunde inhaltsanalytischer Forschung. Z Sexualforsch 2018a; 31: 27-43

Lemke R, Tornow T. Die Darstellung von Sexualität im deutschen Reality-TV: Eine Inhaltsanalyse der Serie „Berlin - Tag und Nacht“. Z Sexualforsch 2018b; 31: 115-133
Lemke R, Weber M. Was wir über die Wirkung von Pornographie wissen (und warum wir vieles nicht wissen). In: Schmidt A, Hrsg. Pornographie. Im Blickwinkel der feministischen Bewegungen, der Porn Studies, der Medienforschung und des Rechts. Baden-Baden: Nomos 2016; $87-122$

Levin RJ. Sexual Activity, Health and Well-Being - The Beneficial Roles of Coitus and Masturbation. Sex Relation Ther 2007; 22: 135-148

Menaker W. Evidence for a Drop in Conceptions in the Big Blackout. American J Obstet Gynecol 1970; 107: 804-806

Müller M. Narrative, Erzählungen und Geschichten des Populismus. Versuch einer begrifflichen Differenzierung. In: Müller M, Precht J, Hrsg. Narrative des Populismus. Wiesbaden: Springer Fachmedien 2019; 1-10

Nielsen RK, Fletcher R, Newman N, Bennen JS, Howard P. Navigating the 'Infodemic': How People in Six Countries Access and Rate News and Information about Coronavirus. Oxford: Reuters Institute for the Study of Journalism 2020 [Als Online-Dokument: https://reutersinstitute. politics.ox.ac.uk/infodemic-how-people-six-countries-access-and-ratenews-and-information-about-coronavirus]

Parmar D, Stavropoulou C, loannidis JPA. Health Outcomes during the 2008 Financial Crisis in Europe: Systematic Literature Review. BMJ 2016; 354: i4588

Prince G. Understanding Narrative. Studies in 20th \& 21st Century Literature 1981; 6: 37-50

Raymond E, Chong E, Winikoff B, Platais I, Mary M, Lotarevich T, Castillo PW, Kaneshiro B, Tschann M, Fontanilla T, Baldwin M, Schnyer A, Coplon L, Mathieu N, Bednarek P, Keady M, Priegue E. TelAbortion: Evaluation of a Direct to Patient Telemedicine Abortion Service in the United States. Contraception 2019; 100: 173-177

[RKI] Robert Koch-Institut. Coronavirus SARS-CoV-2 - SARS-CoV-2 Steckbrief zur Coronavirus-Krankheit-2019 (COVID-19) - 1. Übertragungswege. Berlin: RKI 2020 [Als Online-Dokument: https://www. rki.de/DE/Content/InfAZ/N/Neuartiges_Coronavirus/Steckbrief. html\#doc13776792bodyText1]

Robbins CL, Schick V, Reece M, Herbenick D, Sanders SA, Dodge B, Fortenberry JD. Prevalence, Frequency, and Associations of Masturbation with Partnered Sexual Behaviors among US Adolescents. Arch Pediatr Adolesc Med 2011; 165: 1087-1093

Robertson A. Narrative Analysis. In: Boréus K, Bergström G, Hrsg. Analyzing Text and Discourse. Eight Approaches for the Social Sciences. Los Angeles, CA, London, New Delhi, Singapore, Washington, DC, Melbourne: Sage 2017; 122-145

Rodgers JL, John CAS, Coleman R. Did Fertility Go Up After the Oklahoma City Bombing? An Analysis of Births in Metropolitan Counties in Oklahoma, 1990-1999. Demography 2005; 42: 675-692.

Sandfort A. Coronavirus: Half of Humanity Now on Lockdown as 90 Countries Call for Confinement. EuroNews 03.04.2020 [Als OnlineDokument: https://www.euronews.com/2020/04/02/coronavirus-ineurope-spain-s-death-toll-hits-10-000-after-record-950-new-deathsin-24-hou]

Shaughnessy K, Byers S, Thornton SJ. What Is Cybersex? Heterosexual Students' Definitions. Int J Sex Health 2011; 23: 79-89

Streitmatter R. Sex Sells! The Media's Journey From Repression to Obsession. Cambridge, MA.: Westview Press 2004

Timmermans E, De Caluwé E. Development and Validation of the Tinder Motives Scale (TMS). Comput Human Behav 2017; 70: 341-350

Udry, JR. The Effect of the Great Blackout of 1965 on Births in New York City. Demography 1970; 7: 325-27

Wentland J], Reissing ED. Taking Casual Sex Not Too Casually: Exploring Definitions of Casual Sexual Relationships. Can J Hum Sex 2011; 20: 75-91

[WHO] World Health Organization. Coronavirus Disease 2019 - Coronavirus Disease (COVID-19) Pandemic. Genf: WHO 2020 [Als Online-Dokument: https://www.who.int/emergencies/diseases/novelcoronavirus-2019] 